Document downloaded from:

http://hdl.handle.net/10251/37107

This paper must be cited as:

Jiao, L.; Song, E.; Pla Boscà, VJ.; Li, FY. (2013). Capacity Upper Bound of Channel Assembling in Cognitive Radio Networks with Quasistationary Primary User Activities. IEEE Transactions on Vehicular Technology. 62(4):1849-1855. doi:10.1109/TVT.2012.2236115.

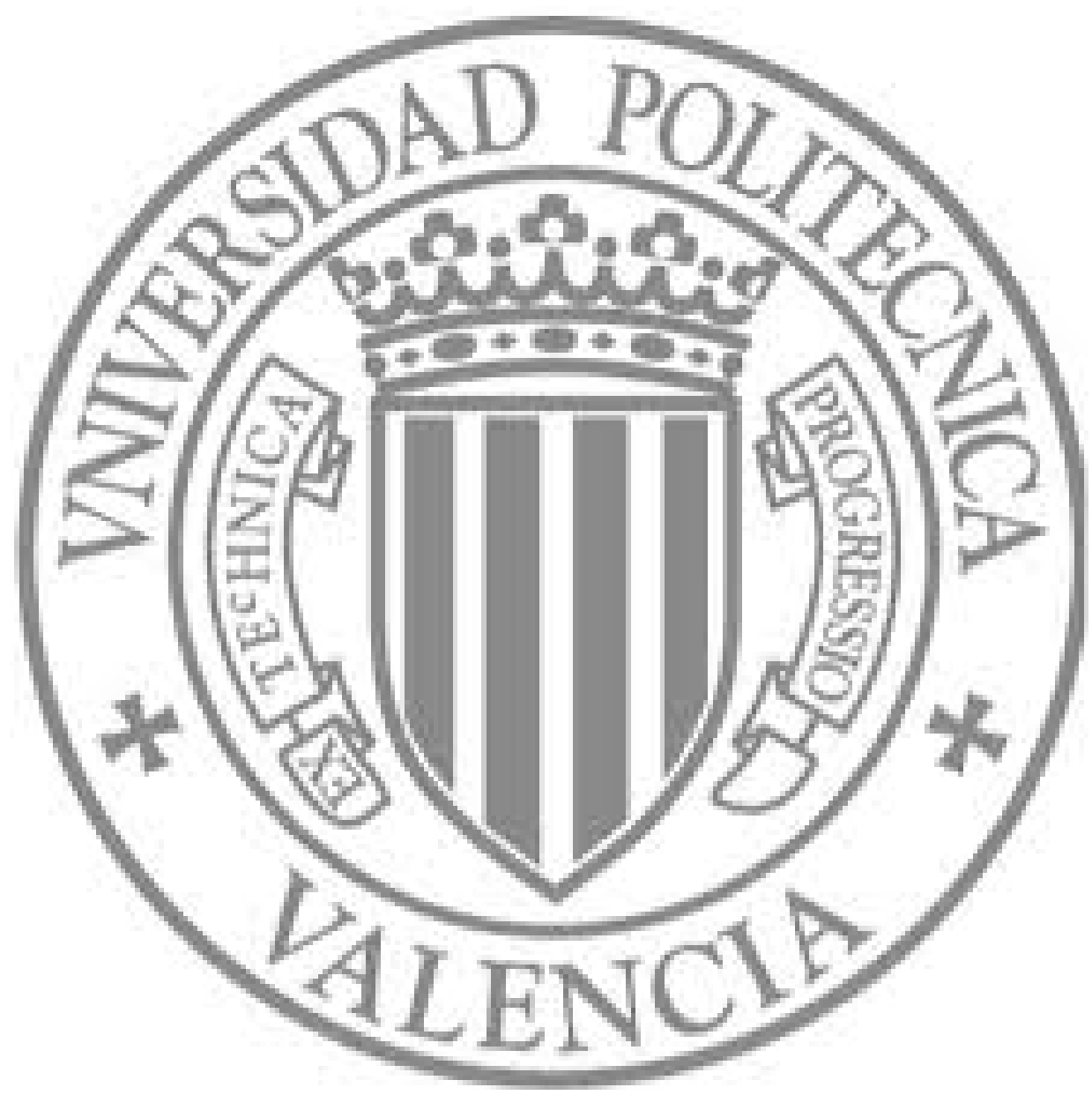

The final publication is available at

http://dx.doi.org/10.1109/TVT.2012.2236115

Copyright Institute of Electrical and Electronics Engineers (IEEE) 


\title{
Capacity Upper Bound of Channel Assembling in Cognitive Radio Networks with Quasistationary Primary User Activities
}

\author{
Lei Jiao, Member, IEEE, Enbin Song †, Vicent Pla, Member, IEEE, \\ and Frank Y. Li, Senior Member, IEEE
}

\begin{abstract}
In cognitive radio networks with multiple channels, various channel assembling strategies may be applied to secondary users, resulting in different achieved capacity. However, there is no previous work on determining the capacity upper bound of channel assembling for secondary users under given system configurations. In this paper, we derive the maximum capacity for cognitive radio networks with channel assembling through Markov chain modeling, considering that PU activities are relatively static compared with $\mathrm{SU}$ services. We first deduce a closed-form expression for the maximum capacity in a dynamic channel assembling strategy, and then demonstrate that no other channel assembling strategy can provide higher capacity than the one achieved by this dynamic strategy.
\end{abstract}

Index Terms-Cognitive radio networks, channel assembling, capacity upper bound, continuous time Markov chain models, quasistationary regime.

\section{INTRODUCTION}

S PECTRUM access schemes in cognitive radio networks (CRNs) [1] can be classified into two categories. The first category is without channel assembling $(\mathrm{ChA})$, i.e., secondary users (SUs) treat each channel as an individual channel [2], [3]. The other one is to assemble several channels together as one channel in order to support SU services with a higher data rate, and this type of ChA strategies has been proposed in many MAC protocols, e.g., [4]-[6]. To further improve system performance, ChA can be integrated with spectrum adaptation (SPA). The meaning of SPA is twofold [7]. Firstly,

Copyright (c) 2012 IEEE. Personal use of this material is permitted. However, permission to use this material for any other purposes must be obtained from the IEEE by sending a request to pubs-permissions @ieee.org.

Manuscript received 26 May 2012, revised 27 September 2012, accepted 24 November 2012

†Corresponding author.

Lei Jiao and Frank Y. Li are with the Dept. of Information and Communication Technology, University of Agder (UiA), N-4898 Grimstad, Norway. E-mail: \{lei.jiao; frank.li\}@uia.no. The work is partially supported by the Research Council of Norway through the ECO-boat MOL project under grant number 210426

Enbin Song is with the Dept. of Mathematics, Sichuan University, 610064 Chengdu, China. E-mail: e.b.song@163.com. The work of this author is supported by the national natural science foundation of China (NSFC) under grant number 60901037, and by the foundation for basic research of Sichuan University for distinguished young scholars 0082604132188.

Vicent Pla is with the Dept. of Communications, Universitat Politècnica de València (UPV), 46022 València, Spain. E-mail: vpla@dcom.upv.es. The work of this author is supported by the Spanish Ministerio de Ciencia e Innovación through the project TIN2010-21378-C02-02.

The cooperation among the authors is supported by the EU Seventh Framework Programme FP7-PEOPLE-IRSES under grant agreement number 247083, project acronym S2EuNet. it supports spectrum handover. Secondly, ongoing SU services can adaptively adjust the number of assembled channels according to the availability of channels as well as other SUs' activities. Without SPA, the number of assembled channels for an ongoing SU service cannot be changed once it is started.

Although various strategies with $\mathrm{ChA}$ have been designed and evaluated by mathematical modeling [7]-[9], how much benefit in terms of capacity ${ }^{1}$ a CRN can achieve at most with ChA is still unknown. In this work, we derive a closed-form expression for the theoretical capacity upper bound (UB) of a secondary network with ChA in the quasistationary regime (QSR). In the QSR, PUs appear sporadically and PU services are long-lasting compared with SU services. The motivation to study the capacity UB in the QSR is that the requirement of relatively static PU activities has been identified as one of the prerequisites that lead to the successful deployment of CRNs [10]. Correspondingly, a number of existing MAC protocols [4], [11] have been designed under this prerequisite. To prove the theoretical UB in the QSR, the capacity UB of a ChA strategy with SPA is derived first, and it is demonstrated thereafter that the capacity of any other strategies will not be higher than this UB in the same regime. Continuous time Markov chain (CTMC) models are utilized for the UB derivation. Note that the derived UB is attainable, meaning that the UB is indeed the maximum capacity for certain strategies. As a result of the mathematical proof, the conditions that a strategy should meet to achieve such maximum capacity are obtained.

The rest of this paper is organized as follows. In Sec. II, the system model is described. In Sec. III, a ChA strategy with SPA is proposed and its maximum capacity is calculated in the QSR. The fact that the capacity of a general strategy cannot exceed the derived maximum capacity in Sec. III is demonstrated in Sec. IV, followed by numerical results and further discussions in Sec. V. Finally, we conclude the paper in Sec. VI.

\section{SYSTEM MODEL}

Consider two types of radios, PUs and SUs, operating in the same spectrum with $M \in \mathbb{N}^{+}$non-overlapping channels for PUs, where $\mathbb{N}^{+}$denotes a set of positive natural numbers. The channels can be utilized by SUs only if they are not

\footnotetext{
${ }^{1}$ Capacity in this context is defined as the average number of service completions per time unit, which is different from the Shannon capacity.
} 
TABLE I

TRANSITIONS FROM A GENERIC STATE $\boldsymbol{x}=(i, j)$ FOR FAFS.

\begin{tabular}{llcl}
\hline Activity & Dest. state & Tran. rate & Conditions \\
\hline PU arrival, no SU forced termination & $(i+1, j)$ & $\lambda_{P}$ & $i<M$ and $M-i-1 \geq j W$. \\
PU arrival, an SU forced termination & $(i+1, j-1)$ & $\lambda_{P}$ & $i<M$ and $M-i-1<j W$. \\
PU departure & $(i-1, j)$ & $i \mu_{P}$ & $i>0$. \\
SU arrival & $(i, j+1)$ & $\lambda_{S}$ & $M-i \geq(j+1) W$. \\
SU departure & $(i, j-1)$ & $\min (M-i, j V) \mu_{S}$ & $j>0$. \\
\hline
\end{tabular}

occupied by PUs. PUs are not aware of the existence of SUs and can access the spectrum at any time. When any PU arrives, SUs must release their occupied channels immediately. Each PU occupies only one channel. However, SUs may assemble multiple channels.

Consider an infrastructure-based CRN, which is composed of a base station, user equipments, and spectrum sensors. The base station is responsible for implementing ChA, SPA, and coordinating sensors for spectrum sensing ${ }^{2}$ [12]. It is assumed that spectrum sensing is performed frequently enough to detect PU's activities promptly with sufficient accuracy. In other words, perfect sensing is achieved from the SU services' perspective. Assume further that the sensing and SPA latency is much shorter than the interval between two consecutive service events, so that service arrival or departure will not happen within the sensing and SPA duration.

As pointed out in [7], only elastic traffic (the traffic type whose service rate will increase if multiple channels are assembled) can obtain benefit in terms of capacity by employing ChA. Therefore, in this paper, we consider elastic traffic type only. Denote by $W$ and $V$ the minimum number and the maximum number of assembled channels in order to support a single SU service respectively, where $1 \leq W \leq V \leq M$, and $W, V \in \mathbb{N}^{+}$. In this expression, $W \geq 1$ means that a service needs at least one channel, and $V \leq M$ means that at most $M$ channels can be assembled by one SU service. The values of $W$ and $V$ reflect the range of the number of channels that can be utilized by an SU service with ChA. In what follows, by selecting a proper strategy and tuning in $W$ and $V$, we will derive the highest possible capacity for a secondary network in the QSR when ChA is enabled. Since our focus is to study the influence of $\mathrm{ChA}$ on capacity, other aspects which can also influence the capacity limit, e.g., queuing and admission control, are beyond the scope of this work.

\section{Strategy Descriptions AND ITs CTMC Model ANALYSIS}

In general, there are two types of $\mathrm{ChA}$ strategies, i.e., dynamic or static ones [7], [9]. The characteristic of a dynamic strategy is that the number of assembled channels for ongoing SU services can be adjusted. However, in a static strategy, the number of assembled channels cannot be changed any longer once a service is started. In what follows, we propose a dynamic strategy, which is different from the dynamic ones proposed in [7] and [9], in order to derive the theoretical capacity UB of CRNs with ChA.

\footnotetext{
${ }^{2}$ Depending on system design, an extra control channel may be required to share control information and exchange sensing results. This possible control channel is not considered as one of these $M$ channels used for data traffic.
}

\section{A. A Strategy with Full Adaptation and Full Channel Sharing}

A dynamic strategy, referred to as full adaptation and full sharing strategy (FAFS), is proposed in this subsection. The strategy has the following features: all the ongoing SU services will always utilize as many channels as possible and they will always equally ${ }^{3}$ share the available channels. Once channels become idle due to a PU or SU service completion or an SU service forced termination, the remaining ongoing SU services will equally share these channels. When a new SU service arrives, it will be allowed to commence if the number of assembled channels per SU service is not fewer than $W$ after accepting the new arrival. Accordingly, when a PU service arrives, ongoing SU services will continue their services but reduce the number of the occupied channels as long as at least $W$ channels are kept for each ongoing SU service after the PU service arrives. However, one ongoing SU service will be forced to terminate if and only if the average number of assembled channels is fewer than $W$ for ongoing SU services after the PU appearance. Obviously, both ChA and SPA are required to support such a strategy.

In the following, we develop CTMCs to model this strategy by assuming that the arrivals of both SU and PU services follow Poisson process with rates $\lambda_{S}$ and $\lambda_{P}$ respectively. Correspondingly, the service times are exponentially distributed with service rates $\mu_{S}$ and $\mu_{P}$ in one channel. Assume further that all channels are homogeneous with the same data rate. Therefore, the service rate of an SU service with $N$ assembled channels is $N \mu_{S}$. The unit for service rate is services/time unit. Given concrete values for these parameters, the capacity can be expressed in kbps or Mbps. For this reason, the unit of capacity is not explicitly expressed in our analysis.

\section{B. The Precise CTMC Model for FAFS}

Let $i$ be the number of ongoing PU services and $j$ be the number of ongoing SU services. The state in the Markov chain of FAFS can be expressed as $(i, j)$, and the transitions from state $(i, j)$ are shown in Table I. Based on these transitions, global balance equations can be established. Then the steady state probability, $\pi(i, j)$, can be calculated based on the balance and the normalization equations. Since there is no assumption on the dynamics of PU services in this model, we refer to this model as precise model.

The capacity of the secondary network based on the precise model, $\rho_{p}$, i.e., the average number of SU service completions

\footnotetext{
${ }^{3}$ To enable equal share, an ongoing SU service is allowed to assemble a non-integer number of channels, as long as this number is in between $W$ and $V$. Non-integer number of channels can be achieved, for example, if a channel can be split into multiple sub-channels and services can access the spectrum on a sub-channel basis using OFDMA technique.
} 
per time unit [13], is given by

$$
\rho_{p}=\sum_{i=0}^{M} \sum_{j=0}^{\lfloor(M-i) / W\rfloor} \min (M-i, j V) \mu_{S} \pi(i, j) .
$$

The blocking probability of SU services, $P_{b}$, is the sum of the probabilities of states that cannot accommodate more SU services, and is expressed by

$$
P_{b}=\sum_{i=0}^{M} \pi(i,\lfloor(M-i) / W\rfloor) .
$$

Forced termination represents a preemption of an ongoing SU service due to PU appearance. Then the forced termination probability is the fraction of forced terminations over commenced SU services, given by

$$
P_{f}=\frac{\lambda_{P}}{\left(1-P_{b}\right) \lambda_{S}} \sum_{j=1}^{\lfloor M / W\rfloor} \pi(M-j W, j) .
$$

\section{The CTMC in the QSR for FAFS}

In the QSR, PU activities are relatively static compared with SU activities. In other words, the distribution of SU services reaches equilibrium between consecutive PU events. In this case, although forced terminations may still occur, there will be, at most, one upon each arrival of PU service. Since between two consecutive arrivals of PUs an arbitrarily large number of SU arrivals occur, the probability of forced termination approaches zero. Therefore, when there are $i$ PU services, the number of available channels is $M-i$ and those channels are in a sense dedicated to $\mathrm{SU}$ services.

Let $\pi(i, j)=\pi(j \mid i) \pi(i)$ be the state probability of the system. The process of PUs can be represented as an Erlang-B model, with state probability $\pi(i)=$ $\left(\frac{\lambda_{P}}{\mu_{P}}\right)^{i} \frac{1}{i !}\left[\sum_{k=0}^{M}\left(\frac{\lambda_{P}}{\mu_{P}}\right)^{k} \frac{1}{k !}\right]^{-1}, 0 \leq i \leq M$. In the QSR, the number of ongoing SU services with $M-i$ dedicated channels (given $i$ ongoing PU services) can be modeled by a birth and death process (BDP) as shown in Fig. 1 , where $Q=M-i$, $I=\left\lfloor\frac{Q}{W}\right\rfloor$ and $C=\left\lfloor\frac{Q}{V}\right\rfloor$. In this BDP, the service rate of the $r$ th state is $r V \mu_{S}$ when $r \leq C$ and $Q \mu_{S}$ when $r>C$.

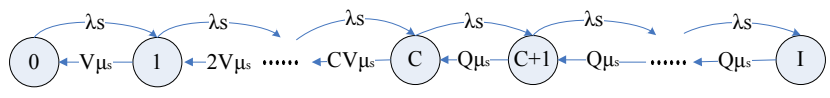

Fig. 1. Illustration of the $\mathrm{BDP}$ for $\mathrm{SU}$ services given $M-i$ dedicated channels.

Fig. 2 illustrates an example from the system point of view in the QSR, when $W=1, V=2$, and $M=3$. The column on the left-hand side indicates the Erlang-B model for PUs. The right-hand side of the figure illustrates the BDPs for the SUs given different instantaneous values of $i$.

Clearly, $\pi(0 \mid i)=1$ if $I=0$ holds. For any $I>0$, with the balance equations, i.e., $\lambda_{S} \pi(0 \mid i)=V \mu_{S} \pi(1 \mid i), \ldots$, $\lambda_{S} \pi(I-1 \mid i)=Q \mu_{S} \pi(I \mid i)$, of the BDPs in Fig. 1, together

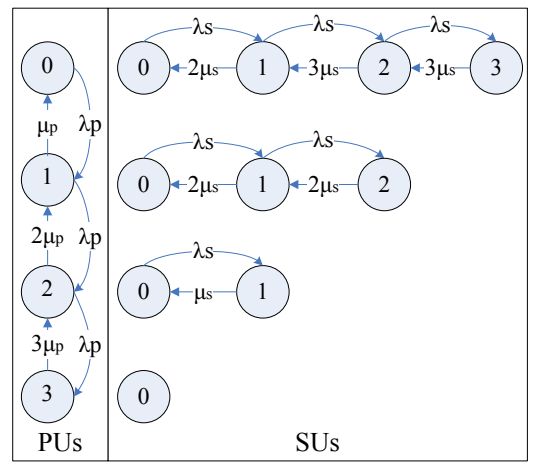

Fig. 2. An exemplary CTMC model in the QSR for FAFS when $W=1$, $V=2$, and $M=3$.

with $\sum_{\forall j \in\{0, \ldots, I\}} \pi(j \mid i)=1$, the state probability is derived as

$$
\begin{aligned}
& \pi(j \mid i)= \begin{cases}\left(\frac{\lambda_{S}}{V \mu_{S}}\right)^{j} \frac{\pi(0 \mid i)}{j !}, & \forall 0 \leq j \leq C, \\
\left(\frac{\lambda_{S}}{Q \mu_{S}}\right)^{j} \frac{Q^{C}}{V C} \frac{\pi(0 \mid i)}{C !}, & \forall C<j \leq I,\end{cases} \\
& \pi(0 \mid i)=\left[\sum_{j=0}^{C}\left(\frac{\lambda_{S}}{V \mu_{S}}\right)^{j} \frac{1}{j !}+\sum_{j=C+1}^{I}\left(\frac{\lambda_{S}}{Q \mu_{S}}\right)^{j} \frac{Q^{C}}{V^{C} C !}\right]^{-1} .
\end{aligned}
$$

Note that the sum of the right-hand expressions in the above balance equations is the capacity of SUs given $i$ PU services, which is expressed as $[1-\pi(I \mid i)] \lambda_{S}$, i.e., the sum of the left-hand expressions in the balance equations. Therefore the capacity in the QSR, $\rho_{q}$ can be expressed as

$$
\rho_{q}=\sum_{i=0}^{M} \pi(i)[1-\pi(\lfloor(M-i) / W\rfloor \mid i)] \lambda_{S} .
$$

In what follows, we give two propositions to acquire the capacity UB of the FAFS in the QSR.

\section{Capacity UB Calculation}

Consider a general BDP with $K+1$ states, where the transition rate from the $(m-1)$ th to the $m$ th state (arrival rate), is $\lambda$, and the transition rate from the $m$ th to the $(m-1)$ th state (service rate), $m \in\{1, \ldots, K\}$ is $\mu_{m}$. Define the average service rate of this process, i.e., capacity, by $\rho^{\prime}=\sum_{i=1}^{K} \pi_{i} \mu_{i}$, where $\pi_{i}$ is the state probability of the $i$ th state.

Proposition 1: With fixed Markov chain length and arrival rate, the average service rate of the BDP, $\rho^{\prime}$, will increase monotonically if $\mu_{i}$ increases, $\forall i \in\{1, \ldots, K\}$.

The proof is deferred to Appendix A. Proposition 1 tells us that with fixed chain length $K+1$ and arrival rate, $\rho^{\prime}$ is maximized if the service rate for each state is maximized. Applying this proposition to the model described in Sec. III-C, with fixed $I, \rho^{\prime}$ will increase when $C$ decreases, and it will reach its maximum value when $C$ is minimized, i.e., $V=M$. Note that $V=M$ indicates that an SU system can utilize all available channels with just one ongoing SU service.

Proposition 2: The average service rate of the BDP will increase if one state (denoted as state $K+1$ ) is added after the last state (denoted as state $K$ ), and the service rate that 
corresponds to the newly added state, $\mu_{K+1}$, satisfies $\mu_{K+1} \geq$ $\max \left\{\mu_{i}\right\}, i \in\{1, \ldots, K\}$.

The proof is deferred to Appendix B. This proposition tells us that higher $\rho^{\prime}$ can be possibly achieved if the Markov chain becomes longer with the service rate of the newly added state not lower than any of the previous service rates in the chain. Applying this proposition to the model described in Sec. III-C, it indicates that higher $\rho^{\prime}$ can be possibly achieved if $I$ is larger, i.e., $W$ is smaller. Therefore, with fixed $C$, the maximum average service rate is achieved when $W=1$.

From Proposition 2, we can also conclude that blocking new arrivals of SU services in order to guarantee minimum bandwidth to protect ongoing SU service cannot provide any benefit in terms of maximizing capacity since the number of ongoing SU services in the system will be lower, i.e., the chain length is reduced.

Considering the results from both propositions, we ascertain that the highest $\rho^{\prime}$ is achieved when $W=1$ and $V=M$ for the BDP for any given $i$. Note that $\rho^{\prime}$ is, indeed, the capacity of SUs given $i$ PU services. Therefore, we can conclude that the maximum capacity of this strategy is achieved when $W=1$ and $V=M$ in the QSR.

The above maximum capacity is derived for a specific strategy, i.e., FAFS. In the next section, we will show the relationship of any other strategies to the FAFS, and conclude that no other strategy can achieve higher capacity than the above maximum capacity by utilizing $\mathrm{ChA}$.

\section{Capacity of General Strategies in the QSR}

For FAFS with $W=1$ and $V=M$, for a given $i$, the BDP achieves the maximum chain length with $M-i+1$ states and the maximum service rate as $(M-i) \mu_{S}$ for each state except state zero. For any other strategy, we can transform its states from its own state space which might be multi-dimensional to a single dimension, i.e., finding an equivalent BDP. We can then compare this BDP with the BDP based on the FAFS in order to check the optimality. By arguing that the chain length and the corresponding service rates in the equivalent $\mathrm{BDP}$ of any other strategy cannot be longer than $M-i+1$ and higher than $(M-i) \mu_{S}, \forall i \in\{0, \ldots, M\}$, we conclude that the maximum capacity based on the FAFS is also the UB for any type of strategy.

Clearly, for any strategy with given $M$ and $i$, the number of ongoing SU services cannot exceed $\lfloor(M-i) / W\rfloor$ and this number reaches its maximum value as $M-i$, when $W=1$. This means that the maximum chain length of the equivalent BDP represented by the number of ongoing SU services in the system cannot exceed $M-i+1$ for any strategy. Note that this maximum chain length, i.e., $M-i+1$, is achieved by the FAFS when $W=1$. Therefore, the chain length of a general strategy cannot be longer than the one achieved by the FAFS with $W=1$, given the same values of $M$ and $i$. To conclude finally that (6) gives the capacity UB, we only need to illustrate that the service rates of the equivalent BDP in a general strategy are lower than or equal to the service rate of the BDP in the FAFS.

Denote by $(i, \phi)$ a general state of a general strategy, where $i$ is the number of PU services while $\phi$ represents the state for
SU services, which might be in multiple dimensions. The state probability of $(i, \phi)$ can be expressed as $\pi(i, \phi)=\pi(\phi \mid i) \pi(i)$, where $\pi(i)$ is the state probability with $i$ PU services. In the QSR, with $i$ ongoing PU services, $\pi(\phi \mid i)$ can be calculated by modeling the SU services with $M-i$ dedicated channels.

Consider a state of SU services, $\phi$, for a general strategy with $M-i$ dedicated channels. Denote by $|\phi|$ the number of ongoing SU services at state $\phi$. Let us re-arrange the states of SU services according to the number of ongoing SU services, i.e., using an integer pair $(r, l)$ to represent a state, $\phi$, where $r$ denotes the number of its ongoing SU services in state $\phi$ and $l$ represents a particular state among all the states that have $r$ SU services. Let $L(r)$ be the number of states that have $r$ SU services. We have $l \in\{1, \ldots, L(r)\}$. Let $\pi^{\prime}(r, l)$ be the state probability of $(r, l)$. Furthermore, let $\zeta_{r}\left(l, l^{\prime}\right)$ be the transition rate from state $(r, l)$ to state $\left(r, l^{\prime}\right), 1 \leq l, l^{\prime} \leq L(r), l \neq l^{\prime}$, considering that in a general strategy channel allocation for SU services could happen even without arrivals or departures of PU or SU services. For a general state, say the $l$ th state with $r$ SU services, denote $b(r, l)$ as the total number of channels that all ongoing SU services assemble at state $(r, l)$. Then the balance equation for the $l$ th state with $r$ services can be expressed as:

$$
\begin{aligned}
& \left(\lambda_{S}+b(r, l) \mu_{S}\right) \pi^{\prime}(r, l)+\pi^{\prime}(r, l) \sum_{l^{\prime}=1, l^{\prime} \neq l}^{L(r)} \zeta_{r}\left(l, l^{\prime}\right) \\
& =\sum_{n=1}^{L(r-1)} P_{r-1, n, l} \lambda_{S} \pi^{\prime}(r-1, n)+\sum_{l^{\prime}=1, l^{\prime} \neq l}^{L(r)} \pi^{\prime}\left(r, l^{\prime}\right) \zeta_{r}\left(l^{\prime}, l\right) \\
& +\sum_{n=1}^{L(r+1)} P_{r+1, n, l}^{\prime} b(r+1, n) \mu_{S} \pi^{\prime}(r+1, n),
\end{aligned}
$$

where $P_{r-1, n, l}$ represents the probability of transition from state $(r-1, n)$ to state $(r, l)$ upon an SU arrival while $P_{r+1, n, l}^{\prime}$ is the probability of transition from state $(r+1, n)$ to state $(r, l)$ upon an SU departure. $P_{r-1, n, l}$ and $P_{r+1, n, l}^{\prime}$ represent different ways of access upon an event in a specific strategy. Note that $\sum_{m=1}^{L(r)} P_{r-1, n, m}=1$ and $\sum_{m=1}^{L(r)} P_{r+1, n, m}^{\prime}=1$.

Let $g(r)=\max _{l}(b(r, l))$. If we sum up all these equations of states with $r$ SU services, (8) holds. Clearly, $\quad \sum_{l=1}^{L(r)} \pi^{\prime}(r, l) \sum_{l^{\prime}=1, l^{\prime} \neq l}^{L(r)} \zeta_{r}\left(l, l^{\prime}\right) \quad=$ $\sum_{l=1}^{L(r)} \sum_{l^{\prime}=1, l^{\prime} \neq l}^{L(r)} \pi^{\prime}\left(r, l^{\prime}\right) \zeta_{r}\left(l^{\prime}, l\right)$. Consequently, we have (9), where $\pi^{\prime \prime}(r)=\sum_{l=1}^{L(r)} \pi^{\prime}(r, l)$.

Now (9) has the same format as the balance equation of the $r$ th state in a BDP. Look at the part that corresponds to the service rate for states with $r$ services on the upper half of (9). Since $\frac{\sum_{l=1}^{L(r)}(g(r)-b(r, l)) \pi^{\prime}(r, l)}{\pi^{\prime \prime}(r)}$ is non-negative, $\left(g(r)-\frac{\sum_{l=1}^{L(r)}(g(r)-b(r, l)) \pi^{\prime}(r, l)}{\pi^{\prime \prime}(r)}\right) \leq g(r)$ holds. Similarly, the part which represents the service rate on the lower half of the equation, i.e., the one that corresponds to states with $r+1$ services, follows the same observation. Obviously, $g(r) \leq M-i$. Therefore, for any ChA strategies, their corresponding SU service rates in their equivalent BDPs are lower than or equal to the service rate of the BDP in the FAFS with $W=1$ and $V=M$, i.e., $(M-i) \mu_{S}$. Recall that the 


$$
\begin{aligned}
& \lambda_{S} \sum_{l=1}^{L(r)} \pi^{\prime}(r, l)+\sum_{l=1}^{L(r)} \pi^{\prime}(r, l) \sum_{l^{\prime}=1, l^{\prime} \neq l}^{L(r)} \zeta_{r}\left(l, l^{\prime}\right)+\mu_{S}\left(g(r)-\frac{\sum_{l=1}^{L(r)}(g(r)-b(r, l)) \pi^{\prime}(r, l)}{\sum_{l=1}^{L(r)} \pi^{\prime}(r, l)}\right) \sum_{l=1}^{L(r)} \pi^{\prime}(r, l)=\lambda_{S} \sum_{n=1}^{L(r-1)} \pi^{\prime}(r-1, n) \\
& +\sum_{l=1}^{L(r)} \sum_{l^{\prime}=1, l^{\prime} \neq l}^{L(r)} \pi^{\prime}\left(r, l^{\prime}\right) \zeta_{r}\left(l^{\prime}, l\right)+\mu_{S}\left(g(r+1)-\frac{\sum_{l=1}^{L(r+1)}(g(r+1)-b(r+1, l)) \pi^{\prime}(r+1, l)}{\sum_{l=1}^{L(r+1)} \pi^{\prime}(r+1, l)}\right)_{l=1}^{L(r+1)} \pi^{\prime}(r+1, l) .
\end{aligned}
$$

$$
\begin{aligned}
& \lambda_{S} \pi^{\prime \prime}(r)+\mu_{S}\left(g(r)-\frac{\sum_{l=1}^{L(r)}(g(r)-b(r, l)) \pi^{\prime}(r, l)}{\pi^{\prime \prime}(r)}\right) \pi^{\prime \prime}(r) \\
& =\lambda_{S} \pi^{\prime \prime}(r-1)+\mu_{S}\left(g(r+1)-\frac{\sum_{l=1}^{L(r+1)}(g(r+1)-b(r+1, l)) \pi^{\prime}(r+1, l)}{\pi^{\prime \prime}(r+1)}\right) \pi^{\prime \prime}(r+1),
\end{aligned}
$$

chain length will not be longer than that in the FAFS given $W=1$ and $V=M$, i.e., the number of ongoing SU services cannot be larger than $M-i$. Based on Propositions 1 and 2, we ascertain that for any strategy with any integer $W$ and $V$ values, its capacity in the QSR will not exceed the capacity in (6) for the FAFS when $W=1$ and $V=M$.

Moreover, from (9), we have $\frac{\sum_{l=1}^{L(r)}(g(r)-b(r, l)) \pi^{\prime}(r, l)}{\pi^{\prime \prime}(r, l)}=0$ when $b(r, l)=g(r), \forall l \in\{1, \ldots, L(r)\}$, meaning that the maximum service rate, i.e., $(M-i) \mu_{S}$, is achievable if $b(r, l)=g(r)=M-i, \forall l \in\{1, \ldots, L(r)\}$. Therefore, if there exists a strategy that fits the following two requirements, the derived capacity UB is also attainable for such a strategy. The first requirement is that the length of the equivalent BDP can reach $M-i+1, \forall i \in\{0, \ldots, M\}$. The other requirement is that for any $i \in\{0, \ldots, M\}$, each of its states with $r$ services is able to utilize $M-i$ channels, i.e., $b(r, l)=M-i$, $\forall l \in\{1, \ldots, L(r)\}$, and $\forall r \in\{1, \ldots, M-i\}$. Example strategies other than the FAFS are the dynamic strategies with $W=1$ and $V=M$ presented in [7], [9]. Note that only dynamic strategies can meet these requirements, since ongoing SU services must be able to adjust their assembled channels adaptively in order to always utilize all channels not occupied by PUs. For static strategies, since ongoing SU services cannot adjust the numbers of their channels, the states that have the same number of ongoing SU services may have different aggregated service rates, resulting in a lower overall service rate in the corresponding BDP.

Furthermore, it is worth mentioning that in the above analyses, $W \geq 1$ is a pre-requisite, i.e., an SU service will utilize at least one channel. More generally, if $W<1$ is allowed, meaning that an SU service may be accommodated with a portion of one channel and that the simultaneous ongoing SU services can be larger than $M$, the capacity UB in the QSR will become larger because the chain length of the corresponding BDP is longer. Still, the capacity UB expression in this case has the same form as (6).

\section{Numerical Results and Further Discussions}

We illustrate the capacity of various strategies derived based on the precise models and the ones in the QSR with given parameters in Fig. 3. More specifically, we plot the numerical results of typical strategies: FAFS with different $W$ and $V$, an example static strategy [7], and the strategy without ChA for comparison. To represent the dynamic feature of PU activities, we introduce a scaler, $f$, to reflect the dynamics of PU activities while keeping the offered load constant for both PUs and SUs, as $\lambda_{P}=f, \mu_{P}=0.5 f, \lambda_{S}=1.5$, and $\mu_{S}=0.82$, given $M=6$. The relationship between the capacity values in the QSR and the results from the precise models can be observed as $f$ varies. When $f \ll 1$, meaning that PUs appear sporadically and PU services are long-lasting, the capacity values from the precise models fit the results in the QSR accuratelly. When $f$ becomes larger, meaning that PUs become dynamic, the capacity values from the precise models deviate more and more from the quasistationary results, due to the fact that more and more SU connections are interrupted as PUs become more active. To reflect other parameters that affect capacity, we illustrate further the results of blocking probability and forced termination probability ${ }^{4}$ as a function of $f$ under the same system configurations in Figs. 4 and 5 respectively.

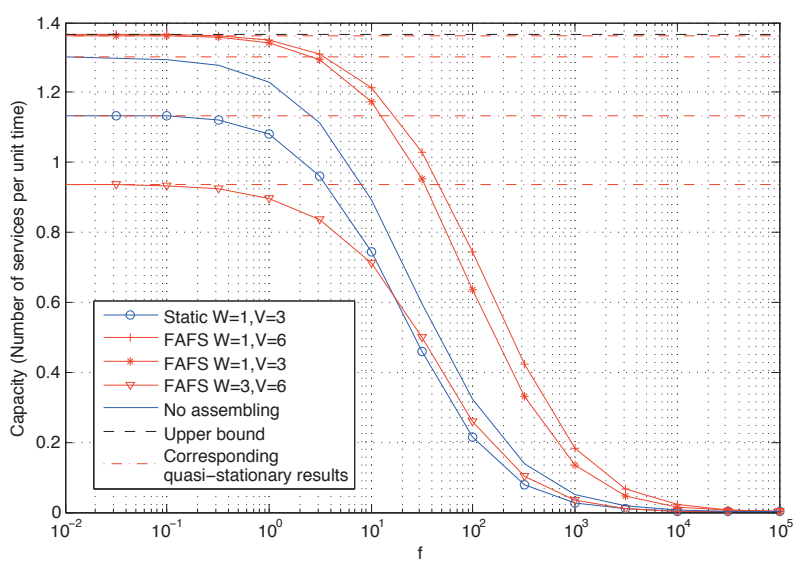

Fig. 3. Capacity of various ChA strategies as a function of $f$.

As can be observed from Fig. 3, the capacity UB in the QSR, i.e., 1.3658, is achieved by FAFS $1 \leq N \leq 6$ when

${ }^{4}$ The relationship among $\rho_{p}, P_{b}$, and $P_{f}$ can be expressed as $\rho_{p}=(1-$ $\left.P_{b}\right)\left(1-P_{f}\right) \lambda_{S}$. This relationship is validated by checking the corresponding values in the curves of Figs. 3-5. These figures illustrate how $\rho_{p}$ is affected by $P_{b}$ and $P_{f}$ in general. However, the capacity upper bound in the QSR is still decided by (6). 


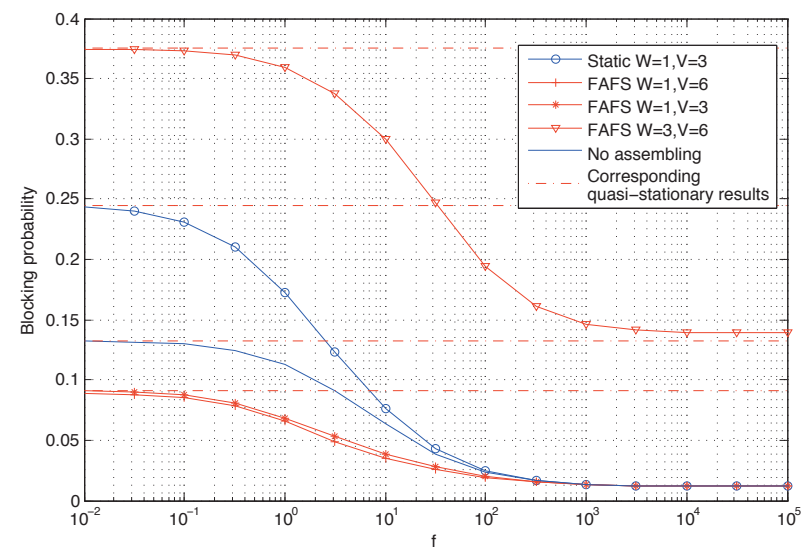

Fig. 4. Blocking probability of various ChA strategies as a function of $f$.

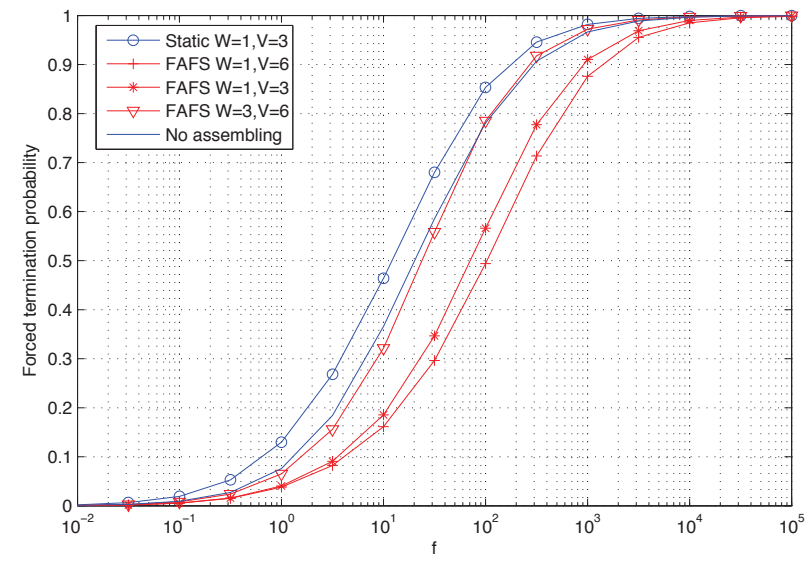

Fig. 5. Forced termination of various ChA strategies as a function of $f$.

$f \ll 1$, followed by FAFS $1 \leq N \leq 3$ with value 1.3635 in the same regime. Other strategies achieve lower capacity in the QSR. Note that although a formal mathematical proof that the capacity under any strategy increases when the dynamics of PUs is scaled down is not available, the claim is intuitively true and it is further supported by the above numerical results. Therefore, we conjecture that the UB obtained in the QSR also applies to a general case when PU activities are dynamic.

\section{CONCLUSions}

Given the arrival and the service rates of $\mathrm{PU}$ and $\mathrm{SU}$ services, a closed-form capacity upper bound of CRNs using channel assembling with quasistationary PU activities has been developed in this paper through CTMC modeling. Regardless of which kind of assembling strategy is adopted, the capacity in CRNs cannot be higher than the value given by (6) with the following parameter configurations: 1) $V=M$ (a single SU service can assemble all channels); and 2) $W$ equals to the lowest possible value, i.e., $W=1$ for $W \in \mathbb{N}^{+}$(the number of channels in order to accommodate a single SU service is set as low as possible). Furthermore, the upper bound is attainable if a dynamic strategy is designed and the above two requirements are satisfied.

\section{APPENDIX A}

PROOF OF PROPOSITION 1

Proof: As discussed in Sec. III-C, $\rho^{\prime}=\left(1-\pi_{K}\right) \lambda$ in the QSR. In the following, we show that $\pi_{K}$ decreases with an increasing $\mu_{i}, i \in\{1, \ldots, K\}$.

From the system balance equations, we have $\pi_{k}=$ $\prod_{i=1}^{k}\left(\lambda / \mu_{i}\right) \pi_{0}, k \in\{1, \ldots, K\}$. Consider the state with $k$ services, $k \in\{1, \ldots, K\}$, we have

$$
\begin{aligned}
1 & =\sum_{i=0}^{K} \pi_{i}=\left[1+\sum_{i=1}^{K} \prod_{j=1}^{i}\left(\frac{\lambda}{\mu_{j}}\right)\right] \pi_{0} \\
& =\left[1+\sum_{i=1}^{k-1} \prod_{j=1}^{i}\left(\frac{\lambda}{\mu_{j}}\right)+\left(\frac{\lambda}{\mu_{k}}\right) \sum_{i=k}^{K} \prod_{j=1, j \neq k}^{i}\left(\frac{\lambda}{\mu_{j}}\right)\right] \pi_{0} \\
& =\left[A+\lambda / \mu_{k} B\right] \pi_{0},
\end{aligned}
$$

where $A=1+\sum_{i=1}^{k-1} \prod_{j=1}^{i} \frac{\lambda}{\mu_{j}}$ and $B=\sum_{i=k}^{K} \prod_{j=1, j \neq k}^{i} \frac{\lambda}{\mu_{j}}$. We can solve (10) for $\pi_{0}$. Note that

$$
\begin{aligned}
\pi_{K} & =\left[\frac{\lambda}{\mu_{k}} \prod_{j=1}^{k-1} \frac{\lambda}{\mu_{j}} \prod_{j=k+1}^{K} \frac{\lambda}{\mu_{j}}\right] \pi_{0}=\frac{\lambda}{\mu_{k}} \prod_{j=1, j \neq k}^{K} \frac{\lambda}{\mu_{j}}\left[A+\frac{\lambda}{\mu_{k}} B\right]^{-1} \\
& =\frac{\lambda \prod_{j=1, j \neq k}^{K}\left(\lambda / \mu_{j}\right)}{A \mu_{k}+B \lambda}
\end{aligned}
$$

It is obvious that (11) is a monotonically decreasing function of $\mu_{k}$. Therefore, the average service rate will increase as $\mu_{k}$ increases monotonically.

\section{APPENDIX B \\ PROOF OF PROPOSITION 2}

Proof: As discussed in Sec. III-C, $\rho^{\prime}=\left(1-\pi_{K}\right) \lambda$ in the QSR. Therefore, we need only to compare probability $\pi_{K}$ in the process before we add the new state and probability $\hat{\pi}_{K+1}$ in the process after the new state is appended. If the proposition is true, $\pi_{K} \geq \hat{\pi}_{K+1}$ must be satisfied, or equivalently,

$$
\frac{1}{1+\sum_{i=1}^{K} \prod_{j=1}^{i} \frac{\lambda}{\mu_{j}}} \geq \frac{\frac{\lambda}{\mu_{K+1}}}{1+\sum_{i=1}^{K} \prod_{j=1}^{i} \frac{\lambda}{\mu_{j}}+\prod_{j=1}^{K+1} \frac{\lambda}{\mu_{j}}}
$$

Obviously, inequality (12) always holds if $\lambda / \mu_{i} \leq 1$. Now consider $\lambda / \mu_{i}>1$. Let $P_{k}=\prod_{i=1}^{k}\left(\lambda / \mu_{i}\right)$. Then inequality (12) becomes $1+P_{1}+\ldots+P_{K} \geq \frac{\lambda}{\mu_{K+1}}\left(1+P_{1}+\ldots+P_{K-1}\right)$, which can be rewritten as

$$
\begin{aligned}
& 1+\left(P_{1}-\frac{\lambda}{\mu_{K+1}}\right)+\left(P_{2}-P_{1} \frac{\lambda}{\mu_{K+1}}\right) \\
& \ldots+\left(P_{K}-P_{K-1} \frac{\lambda}{\mu_{K+1}}\right) \geq 0 .
\end{aligned}
$$

Since $\mu_{K+1} \geq \max \left\{\mu_{i}\right\}, i \in\{1, \ldots, K\}$, therefore $P_{1} \geq$ $\lambda / \mu_{K+1}$ and $P_{k+1} \geq P_{k}\left(\lambda / \mu_{K+1}\right), \forall k \in\{1, \ldots, K-1\}$. Hence inequality (13) holds, and consequently, $\pi_{K} \geq \hat{\pi}_{K+1}$ holds. 


\section{REFERENCES}

[1] Y. C. Liang, K. C. Chen, G. Y. Li, and P. Mahönen, "Cognitive radio networking and communications: An overview," IEEE Trans. Veh. Technol., vol. 60, no. 7, pp. 3386-3407, Sep. 2011.

[2] S. Huang, X. Liu, and Z. Ding, "Optimal transmission strategies for dynamic spectrum access in cognitive radio networks," IEEE Trans. Mobile Comput., vol. 8, no. 12, pp. 1636-1684, Nov. 2009.

[3] J. Martinez-Bauset, V. Pla, J. R. Vidal, and L. Guijarro, "Approximate analysis of cognitive radio systems using time-scale separation and its accuracy," IEEE Commun. Lett., 2012. DOI: 10.1109/LCOMM.2012.111612.121490.

[4] IEEE standard for wireless regional area networks-part 22: Cognitive wireless RAN medium access control (MAC) and physical layer (PHY) specifications: Policies and procedures for operation in the TV bands, IEEE Std. 802.22WG, Jul. 2011.

[5] J. Jia, Q. Zhang, and X. Shen, "HC-MAC: A hardware-constrained cognitive MAC for efficient spectrum management," IEEE J. Select. Areas Commun., vol. 26, no. 1, pp. 106-117, Jan. 2008

[6] H. A. B. Salameh, M. M. Krunz, and O. Younis, "MAC protocol for opportunistic cognitive radio networks with soft guarantees," IEEE Trans. Mobile Comput., vol. 8, no. 10, pp. 1339-1352, Oct. 2009.

[7] L. Jiao, F. Y. Li, and V. Pla, "Modeling and performance analysis of channel assembling in multi-channel cognitive radio networks with spectrum adaptation," IEEE Trans. Veh. Technol., vol. 61, no. 6, pp. 2686-2697, Jul. 2012.

[8] J. Lee and J. So, "Analysis of cognitive radio networks with channel aggregation," in Proc. IEEE WCNC, Sydney, Australia, Apr. 2010.

[9] L. Jiao, F. Y. Li, and V. Pla, "Dynamic channel aggregation strategies in cognitive radio networks with spectrum adaptation," in Proc. IEEE GLOBECOM, Houston, TX, USA, Dec. 2011.

[10] C. Politis, "Managing the radio spectrum: the role of cognitive radio in future spectrum use," IEEE Vehicular Technology Magazine, vol. 4, no. 1, pp. 20-26, Mar. 2009.

[11] Y. Yuan, P. Bahl, R. Chandra, P. A. Chou, J. I. Ferrell, T. Moscibroda, S. Narlanka, and Y. Wu, "KNOWS: Cognitive radio networks over white spaces," in Proc. IEEE DySPAN, Dublin, Ireland, Apr. 2007.

[12] T. Yucek and H. Arslan, "A survey of spectrum sensing algorithms for cognitive radio applications," IEEE Commun. Surveys Tuts., vol. 11, no. 1, pp. 116-130, First Quarter 2009.

[13] X. R. Zhu, L. F. Shen, and T. S. P. Yum, "Analysis of cognitive radio spectrum access with optimal channel reservation," IEEE Commun. Lett., vol. 11, no. 4, pp. 304-306, Apr. 2007. 